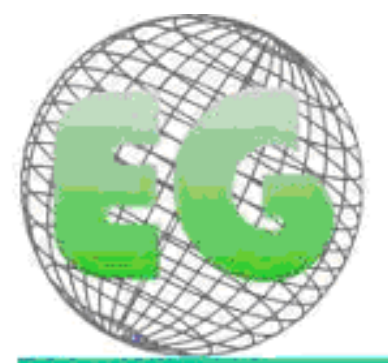

ISSN 1696-6) सक

\title{
CALIDAD DE VIDA Y NECESIDADES DE FORMACIÓN DE LAS CUIDADORAS FORMALES DE PERSONAS MAYORES DEPENDIENTES INSTITUCIONALIZADAS
}

LIFE QUALITY AND TRAINING NEEDS FOR THE FORMAL CARE GIVERS OF DEPENDENT ELDERLY INSTITUTIONALIZED \begin{abstract}
${ }^{* *}$ Puig Llobet, M, **Lluch Canut, MT, ${ }^{* * *}$ Sabater Mateu, P., ${ }^{* * * *}$ Rodríguez Ávila, N.
*Profesora Asociada. Departamento de Enfermería de Salud Pública, Salud Mental y Materno-Infantil. Escuela de Enfermería. ${ }^{* *}$ Catedrática de Enfermería Psicosocial y Salud Mental. Departamento de Enfermería de Salud Pública, Salud Mental y Materno-Infantil. Escuela de Enfermería. ${ }^{* * *}$ Profesora Titular. Departamento de Enfermería de Salud Pública, Salud Mental y Materno-Infantil. Escuela de Enfermería. ${ }^{* * * *}$ Doctora en Sociología. Profesora. Departamento de Sociología y Análisis de las Organizaciones. Facultad de Economía y
\end{abstract} Empresa. Universidad de Barcelona

Palabras clave: Auxiliar de geriatría, satisfacción con la vida, calidad de vida, dependencia, formación, institucionalización, mayores dependientes, cuidados.

Keywords: Life Satisfaction, Life Quality, Dependency, Training, Institutionalization, Dependant Elderly, and Care.

\section{RESUMEN}

Objetivo: Identificar la calidad de vida, satisfacción personal y laboral de las Auxiliares de Geriatría cuidadoras de personas mayores institucionalizadas. Metodología: Estudio descriptivo realizado a partir de datos cuantitativos y datos cualitativos. El tamaño de la muestra fue de 12 Auxiliares de Geriatría que trabajan en el Centro Sociosanitario Ricard Fortuny y en la Residencia Sant Francesc de Vilafranca del Penedès. Resultados: La mayoría está satisfecha con su vida personal y tienen una buena calidad de vida. Les gusta su trabajo y están motivadas, sin embargo, manifiestan estar poco valoradas y remuneradas, piensan que la formación es importante para ofrecer unos cuidados de calidad. Conclusiones y discusión: Se necesita una formación especializada para ofrecer unos cuidados de calidad a los mayores institucionalizados, ello no sólo influye en la satisfacción laboral de las Auxiliares de Geriatría, sino también en su satisfacción personal y calidad de vida. 


\section{ABSTRACT}

Objective: To identify the life quality and personal and job satisfaction of geriatrics assistants, caregivers of the institutionalized elderly. Methodology: Descriptive study made from quantitative and qualitative data. The sample size was 12 geriatrics assistants that work in the center Sociosanitario Ricardo Fortuny and in Sant Francesc de Vilafranca del Penedès Residence. Results: Most of them are satisfied with their personal life and have good life quality. They like their job and are motivated. However, they stated being little appreciated and badly paid. They think training is important to deliver quality care. Conclusion and discussion: Specialized training is needed to deliver quality care to the institutionalized elderly, as it does not only affect the job satisfaction of geriatrics assistants but also their personal satisfaction and quality of life.

\section{INTRODUCCIÓN}

El envejecimiento de la población implica el aumento del número de personas que padecen enfermedades crónicas y presentan situaciones de dependencia, sobre todo el colectivo de personas de 75 y más años $(1,2)$. Ello implica la necesidad de formar profesionales que puedan atender las necesidades de estas personas, tanto a nivel domiciliario como a nivel institucional. El cuidar de los mayores ha sido visto como una tarea fácil, sin ser necesario el tener una formación especializada (3), en parte, porque cuidar forma parte de las tareas atribuidas a las mujeres (4) y también por el valor social atribuido a los mayores (5). Sin embargo, algunos de los cuidados que reciben las personas mayores dependientes requieren ciertas habilidades y una formación adecuada, como por ejemplo, el evitar úlceras por presión o el estimular actividades que favorezcan la reminiscencia (6). Existen distintos factores que influyen en los cuidados, tales como la motivación en el trabajo, la satisfacción personal, la calidad de vida (7) y el nivel de formación de los profesionales.

La calidad de vida es un constructo tratado desde muchas perspectivas y donde no hay acuerdo unánime (8). Tener y percibir una buena calidad de vida está relacionado con distintas situaciones personales; en este estudio se realiza un enfoque subjetivo de la calidad de vida que ligamos a distintas dimensiones relacionadas con la satisfacción laboral, estableciendo un marco conceptual que puede ser útil como evaluación de la satisfacción personal versus satisfacción laboral, relacionando estos factores con la realización de unos cuidados de calidad (Gráfico 1).

Gráfico 1: Dimensiones relacionadas con la calidad de los cuidados

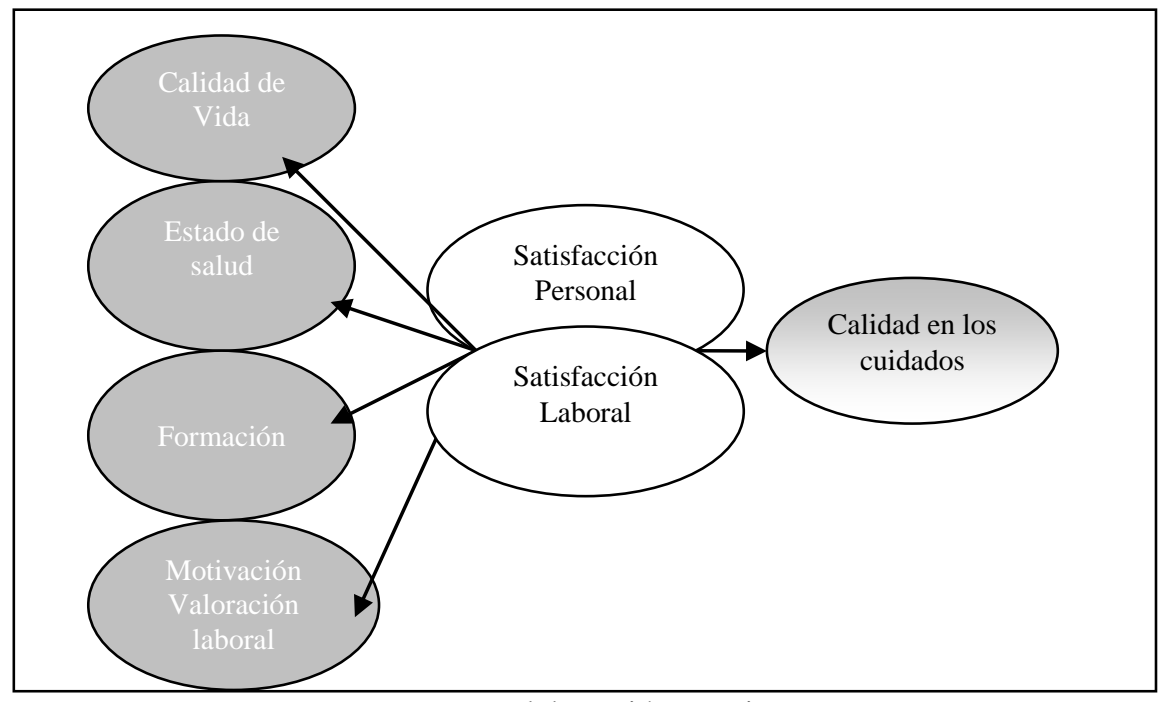

Fuente: elaboración propia 
Respecto a la calidad de vida, nos basamos en la concepción que tiene el sujeto en torno al propio constructo, a la percepción de la calidad de vida y felicidad y al grado de satisfacción personal. También se incluye la percepción del estado de salud, valorando que es importante cuidarse para poder cuidar mejor. La necesidad de formación es otro elemento importante, así como la motivación laboral, la percepción en la valoración y remuneración laboral y la satisfacción en el trabajo realizado. Por esta razón, nos hemos planteado el objetivo de explorar los elementos que influyen en la calidad de vida, satisfacción personal y laboral de las Auxiliares de Geriatría que cuidan a personas mayores institucionalizadas. Concretamente pretendemos: a) identificar la calidad de vida y satisfacción personal; b) analizar la percepción del estado de salud; c) determinar las necesidades de formación y d) valorar la motivación y la satisfacción laboral.

\section{MATERIAL Y MÉTODOS}

Se trata de un estudio descriptivo realizado a partir de datos cuantitativos y datos ualitativos. El tamaño de la muestra fue de 12 Auxiliares de Geriatría que trabajan en el Centro Sociosanitario Ricard Fortuny y en la Residencia Sant Francesc de la ciudad de Vilafranca del Penedès. Todas las cuidadoras eran mujeres, con una media de edad de 45 años. En general, realizaban una jornada laboral de ocho horas diarias. Para participar en el estudio, se incluyeron a todas las Auxiliares que asistieron a las clases presénciales de un curso formativo realizado en el Centro de Estudios CET Penedès de la ciudad de Vilafranca del Penedès y que quisieron participar en el estudio.

Las variables estudiadas fueron: a) satisfacción con la vida personal; b) percepción de felicidad; c) calidad de vida percibida; d) cómo conciben la calidad de vida, e) percepción del estado de salud; f) importancia de tener formación para cuidar de los mayores; g) motivación al realizar su trabajo; h) percepción de valoración y remuneración de su trabajo; i) satisfacción laboral y k) datos sociodemográficos. Las variables definidas fueron evaluadas a partir de un cuestionario de elaboración propia (tabla I).

Tabla I: Cuestionario de evaluación de la percepción subjetiva de las dimensiones de calidad de vida, estado de salud, formación, motivación y satisfacción laboral en Auxiliares de Geriatría que trabajan en Centros Sociosanitarios

\begin{tabular}{|c|c|c|}
\hline Apartados & Variables & Ítems \\
\hline $\begin{array}{l}\text { A. Calidad de vida, } \\
\text { satisfacción personal y } \\
\text { felicidad }\end{array}$ & $\begin{array}{l}\text { A.1. ¿Está satisfecha con su } \\
\text { vida? } \\
\text { A.2. ¿Se siente feliz? } \\
\begin{array}{l}\text { A.3. ¿Cómo percibe su calidad } \\
\text { de vida? }\end{array} \\
\text { A.4. ¿Como concibe la calidad } \\
\text { de vida? }\end{array}$ & $\begin{array}{l}\text { 1. Muy satisfecha. 2. Bastante } \\
\text { satisfecha. 3. Poco satisfecha. } 4 . \\
\text { Nada satisfecha. } \\
\text { 1. Muy feliz. 2. Bastante feliz. } 3 . \\
\text { Poco feliz. 4. Nada feliz. } \\
\text { 1. Muy buena. 2. Buena. } 3 . \\
\text { Regular. 5. Mala. 6. Muy mala. } \\
\text {---------- }\end{array}$ \\
\hline B. Percepción estado de salud & $\begin{array}{l}\text { B.1. ¿Cómo percibe su estado } \\
\text { de salud? }\end{array}$ & $\begin{array}{l}\text { 1. Muy bueno. 2. Bueno. } 3 \text {. } \\
\text { Regular. 5. Malo. 6. Muy malo. }\end{array}$ \\
\hline
\end{tabular}




\begin{tabular}{|l|l|l|}
\hline C. Formación & $\begin{array}{l}\text { C.1. ¿Piensa que es importante } \\
\text { tener formación para cuidar a } \\
\text { los mayores } \\
\text { institucionalizados? }\end{array}$ & 1. Sí. 2. No. \\
\hline \begin{tabular}{l|l|l|} 
D. Motivación y Satisfacción \\
laboral
\end{tabular} & $\begin{array}{l}\text { D.1. ¿Está motivada a la hora } \\
\text { de realizar los cuidados del } \\
\text { mayor? }\end{array}$ & 1. Sí. 2. No. \\
& $\begin{array}{l}\text { D.2 ¿Cree que el colectivo de } \\
\text { profesionales de geriatría está } \\
\text { valorado? }\end{array}$ & 1. Sí. 2. No. \\
& $\begin{array}{l}\text { D3. ¿Cree que el colectivo de } \\
\text { profesionales de geriatría está } \\
\text { bien remunerado? }\end{array}$ & 1. Sí. 2. No. \\
\hline E. Datos sociodemográficos & $\begin{array}{l}\text { E1.Sexo. ¿Está satisfecha con su } \\
\text { trabajo? }\end{array}$ & 1. Si 2. No. Edad. \\
\hline
\end{tabular}

Fuente: elaboración propia.

El cuestionario autoadministrado fue pasado durante el transcurso de un curso de formación realizado en el Centro de Estudios CET Penedès durante el año académico 2007-2008. Consta de una pregunta abierta para valorar como conciben las auxiliares la calidad de vida, cuatro preguntas con una escala tipo liker para valorar la satisfacción con la vida, la percepción de felicidad, la percepción de calidad de vida y la percepción del estado de salud y cinco preguntas dicotómicas para determinar la importancia de la formación, la percepción de motivación laboral, la opinión de la valoración, remuneración y satisfacción laboral. También se han incluido ciertos datos sociodemográficos. Los datos se analizaron mediante el programa estadístico SPSS 16. Se realizaron análisis descriptivos de frecuencias y porcentajes para los datos cuantitativos. Los datos cualitativos se agruparon por análisis de contenido y agrupación por categorías.

\section{RESULTADOS}

En relación a la satisfacción con la vida el 66,7\% (n: 8) describe estar bastante satisfecha con su vida y en menor proporción, el 33,3\% (n: 4) están muy satisfechas con su vida personal. Respecto a la percepción de felicidad, tal como se observa en la tabla II, un $75 \%$ se muestra bastante feliz.

Tabla II: Percepción de felicidad

\begin{tabular}{lcc}
\hline Variables & $\%$ & $\mathrm{n}$ \\
\hline Muy feliz & 16,7 & 2 \\
Bastante feliz & 75,0 & 9 \\
Poco feliz & 8,3 & 1 \\
Total & 100 & 12 \\
\hline
\end{tabular}


Su calidad de vida actual es considerada buena por un $75 \%$ (n: 9) de las cuidadoras, y un $25 \%(n: 3)$ la considera muy buena. En relación a cómo conciben la calidad de vida, las respuestas se engloban en tres dimensiones: Bienestar físico (autonomía y salud), bienestar psíquico (satisfacción con la vida y vivir sin estrés) y Recursos (dinero y recursos para cubrir las necesidades). Un $92 \%$ (n: 11) perciben un buen estado de salud (tabla III).

Tabla III: Percepción del estado de salud

\begin{tabular}{lll}
\hline Variables & $\%$ & $\mathrm{n}$ \\
\hline Muy Bueno & 25 & 3 \\
Bueno & 66,7 & 8 \\
Regular & 8,3 & 1 \\
\hline Total & 100 & 12 \\
\hline
\end{tabular}

Tener formación es importante para todas las entrevistadas, además lo ven necesario para proporcionar unos cuidados de calidad. Por ello, agradecen a su institución la oportunidad de realizar cursos de formación. En relación a la motivación laboral, el $75 \%$ ( $\mathrm{n}$ : 9) se muestran motivadas al realizar su trabajo, sin embargo todas las cuidadoras piensan que este colectivo está poco valorado y remunerado profesionalmente, pero se muestran satisfechas realizando su trabajo. Proponen mejorar las condiciones laborales de este colectivo, respecto a horarios, remuneración y valoración profesional.

\section{DISCUSIÓN Y CONCLUSIONES}

Hay un acuerdo de todas las Auxiliares de Geriatría del estudio en considerar la formación como elemento importante a la hora de aplicar unos cuidados de calidad a los mayores institucionalizados que atienden. Ello pone de manifiesto, que es necesario tener una formación especializada en geriatría y gerontología, a poder ser, reglada, para el cuidado de mayores que requieren institucionalización o cuidados complejos. Sin embargo, hay la tendencia a pensar que existen situaciones que se solventan por el hecho de ser mujer y tener socializado el cuidar (6). Es por ello, que las instituciones sanitarias deberían fomentar la formación de las personas que contratan para cuidar de los mayores institucionalizados. Así como evitar que estos cuidadores asuman responsabilidades de las que no tienen competencia, como por ejemplo administrar inyecciones subcutáneas (9).

Estas cuidadoras perciben una buena calidad de vida y están satisfechas con su vida, se sienten felices y conciben la calidad de vida en tener bienestar físico, psíquico y recursos necesarios para satisfacer las necesidades. El estado de salud percibido es considerado bueno por la mayoría de las auxiliares del estudio. El sentirse bien y tener salud contribuye a la satisfacción personal y saberse cuidar favorece el cuidado de las personas que se tienen a cargo (10). Respecto a la satisfacción y motivación laboral, estas auxiliares se muestran en su mayoría motivadas y satisfechas con su trabajo, a pesar que reconocen que este colectivo está mal remunerado y valorado socialmente, situación identificada en otro estudio (11). Mejorar las condiciones laborales de este colectivo podría ayudar a mejorar la autoestima de este colectivo y contribuir a mejorar la calidad y cuidados aplicados a las personas mayores. Por último, insistir que las personas mayores se merecen unos cuidados profesionales y de calidad cuando sus necesidades lo requieren, tanto a nivel domiciliario como a nivel institucional. Y los profesionales que cuidan de estos mayores, necesitan unas mejores condiciones formativas y laborales, ello no sólo influye en su satisfacción laboral, sino también en su satisfacción personal y percepción de calidad de vida. 


\section{Agradecimientos:}

Al Centro de Estudios CET Penedès y a las cuidadoras profesionales entrevistadas.

\section{BIBLIOGRAFÍA}

1. Instituto de Mayores y Servicios Sociales. Bases Demográficas. En Libro Blanco Atención a las personas en situación de dependencia en España. Madrid: IMSERSO, 2005.

2. Castejón, Penélope; Abellán, Antonio. Estado de Salud. En: Informe 2006. Las personas mayores en España. Observatorio de Personas Mayores. Madrid: IMSERSO, 2006.

3. Instituto de Mayores y Servicios Sociales. Cuidados a las personas mayores en los hogares españoles: El entorno familiar. Colección Estudios Serie Dependencia. Madrid:

IMSERSO, 2005.

4. Campo Ladero, Maria José. Apoyo informal a las personas mayores y el papel de la mujer Cuidadora. Opiniones y Actitudes nํ 31. Madrid: Centro de Investigaciones Sociológicas (CIS), 2000.

5. Losada Baltar, Andrés. Edadismo: consecuencias de los estereotipos, del prejuicio y la discriminación en la atención de las personas mayores. Algunas pautas para la intervención. (Madrid, España). Portal Mayores, Informes Portal Mayores, nº 14. 2004. Disponible en: http://www.imsersomayores.csic.es/documentos/documentos/losada-edadismo-pdf [Consultado el 12.6.2009].

6. Instituto de Mayores y Servicios Sociales. Consideraciones en torno a la protección de las personas en situación de dependencia. En Libro Blanco Atención a las personas en situación de dependencia en España. Madrid: IMSERSO, 2005.

7. Sánchez González, R; Álvarez Nido, R; Lorenzo Borda, S 2003, 13:4: 55-60. Calidad de vida profesional de los trabajadores de Atención Primaria del Área 10 de Madrid (Madrid, España). Medifam. [online].

Disponible en: <http://scielo.isciii.es/scielo.php?script=sci_arttext\&pid=S1131-

57682003000400009\&lng=es\&nrm=iso >[Consultado el 15.7.2009].

8. Gómez Vela, M; Sabeh, E. Calidad de vida. Evolución del concepto y su influencia en la investigación y la práctica. 2006. Disponible en:

http://www3.usal.es/inico/investigacion/invesinico/calidad.htm.[Consultado el 18.7.2009].

9. Corpas F. Limitación de las funciones de los gerocultores. Enfermería Facultativa 2009. XVIII Nueva Época: (23):30.

10. Novel Martí, Gloria; Lluch Canut, María Teresa; Miguel López de Vergara, Maria Dolores. Enfermería psicosocial y Salud Mental. Barcelona: Ed. Masson, 2005. 11. La situación actual de las residencias en Geriatri-k. Revista profesional del sector asistencial geriátrico, 2006. Disponible en http://www.geriatrik.com/index.php?option=com content\&task=view\&id=78\&ltemid=48 [Consultado el 12.6.2009]. 